\title{
Systematic review of statins in sepsis: There is no evidence of dose response
}

\author{
Morgan Quinn', Claire Moody¹, Bill Tunnicliffe', Zahid Khan'1, Mav Manji', Sandeep Gudibande', \\ Nick Murphy ${ }^{1}$, Tony Whitehouse ${ }^{1}$, Catherine Snelson ${ }^{1}$, Tonny Veenith ${ }^{1,2,3}$
}

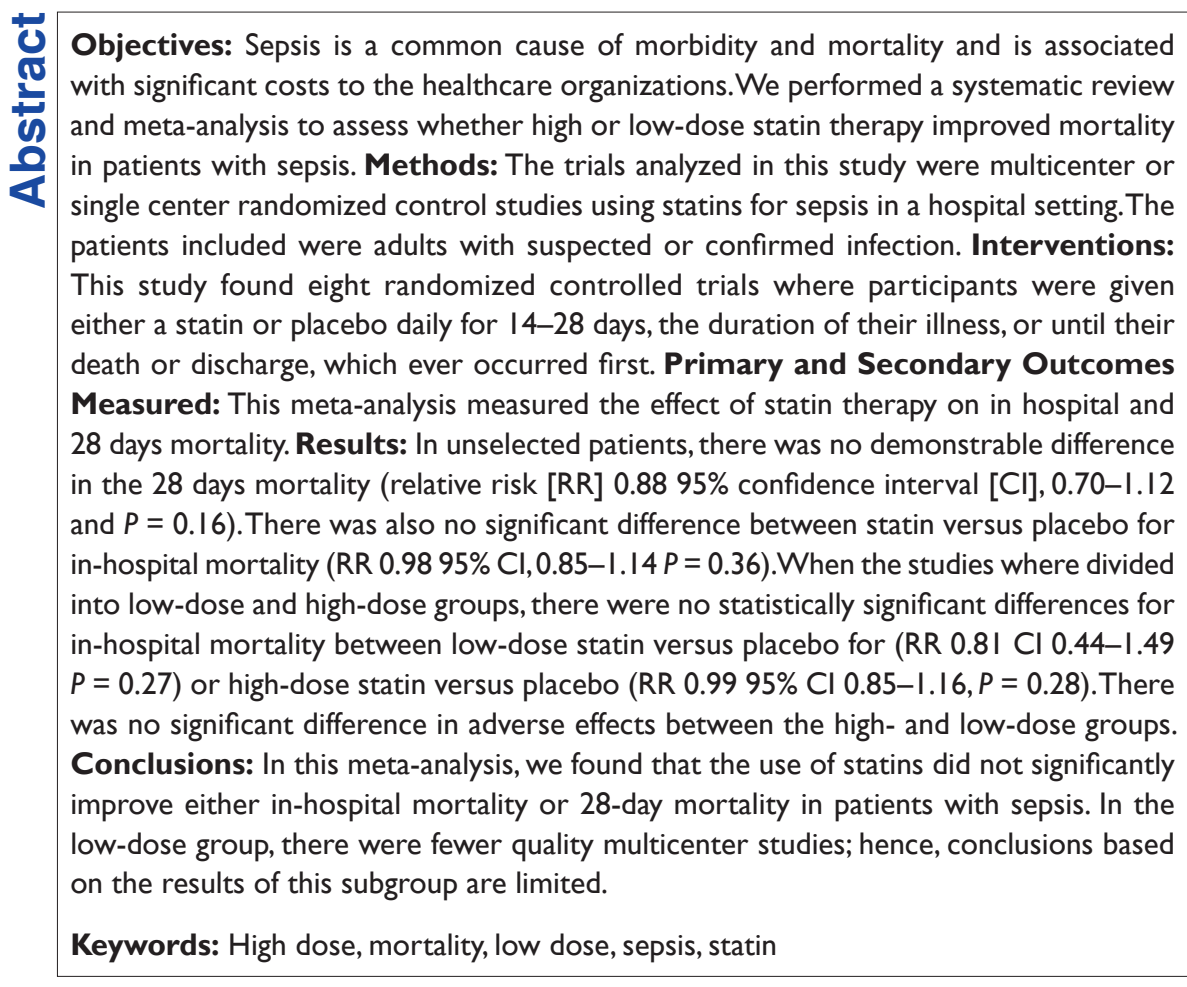

\begin{tabular}{|l|}
\hline Access this article online \\
\hline Website: www.ijccm.org \\
\hline DOI: 10.4103/0972-5229.190366 \\
\hline Quick Response Code: \\
\hline
\end{tabular}

\section{Introduction}

Sepsis is characterized by an exaggerated inflammatory response to infection, resulting in a multisystem physiological, cellular, and organ dysfunction. In a European study, a typical episode of severe sepsis costs the health care organization approximately $€ 25,000$. $^{[1]}$

\section{From:}

'Department of Critical Care, Queen Elizabeth Medical Centre, Birmingham, B15 2TH, '2Division of Anaesthesia, University of Cambridge, Addenbrooke's Hospital, Cambridge, CB2 OQQ, ${ }^{3}$ School of Immunity and Infection, University of Birmingham, B15 2TT, UK

\section{Correspondence:}

Dr. Tonny Veenith, Department of Critical Care,

Queen Elizabeth Medical Centre, Birmingham,

B15 2TH, UK.

E-mail: tv227@cam.ac.uk
Assuming that we see 100,000 cases of severe sepsis per annum, this equates to a direct current cost to the national health services of England of over 2.5 billion every year. In the USA, the Centers for Disease Control and Prevention's National Center for Health Statistics estimates the number of hospital admissions attributed

This is an open access article distributed under the terms of the Creative Commons Attribution-NonCommercial-ShareAlike 3.0 License, which allows others to remix, tweak, and build upon the work non-commercially, as long as the author is credited and the new creations are licensed under the identical terms.

For reprints contact: reprints@ medknow.com

How to cite this article: Quinn M, Moody C, Tunnicliffe B, Khan Z, Manji M, Gudibande $\mathrm{S}$, et al. Systematic review of statins in sepsis: There is no evidence of dose response. Indian J Crit Care Med 2016;20:534-41. 
to sepsis increased from 621,000 in the year 2000 to $1,141,000$ in $2008 .{ }^{[2]}$ In 2011, sepsis resulted in an aggregate healthcare cost of $\$ 20.3$ billion making it the most expensive condition treated in US hospitals. ${ }^{[3]}$

Mortality from sepsis has improved over the last decade but is still estimated to be $36 \%$ in Europe. ${ }^{[1]}$ This warrants a search for novel therapeutic targets and preventative therapies in patient with sepsis. Statins are lipid-lowering drugs that inhibit 3-hydroxy-3 methyglutaryl coenzyme A reductase, with pleotropic mechanisms that may be beneficial in sepsis. They are hypothesized to possess a variety of benefits including anti-inflammatory, immunomodulatory, antioxidant, and antithrombotic effects. ${ }^{[4,5]}$ In previous studies, statins have been shown to reduce the unregulated immune response, influence the gene transcription, and reduce the expression of mRNA to sepsis. The use of statins was associated with an improved mortality and morbidity following sepsis. ${ }^{[6,7]}$ In animal studies, statins reduced the severity of sepsis. ${ }^{\left[{ }^{[8,9]}\right.}$ It is also thought that statins exert a protective effect through down-regulation of toll-like receptor 4, inhibition of nuclear factor-kappa-B and protection against endothelial cell apoptosis. ${ }^{[10,11]}$ Despite the initial promise from animal data and early clinical trials, these benefits have not been reproduced in the large, well-designed, randomized controlled trials (RCTs) published recently. ${ }^{[12,13]}$

This paper aims to assess the impact of both high and low dose statin therapy on in-hospital and 28-day mortality in patients with sepsis.

\section{Methods}

\section{Search strategy for published studies with aggregate data}

Electronic databases, including PubMed and EMBASE, were searched using a combination of keywords: "sepsis," "intensive care" "statin" "simvastatin," "atorvastatin," and "rosuvastatin" to create a list of articles published before October 2015. The search was limited to articles published in the English language.

\section{Study selection criteria}

Articles were included if the study population was adult patients with a suspected or confirmed infection and reported 28 day or in-hospital mortality reported as a primary or secondary outcome. A total of 215 articles were identified. The following were excluded; 160 were not clinical trials, 2 post hoc analyses, 2 cell-based studies, 3 were not randomized control trials, and 31 were the wrong patient population. A total of 15 studies were fully assessed for eligibility. Of these, 3 were excluded as mortality was not reported as a primary or secondary outcome, and 4 were excluded as they were the wrong patient populations: 3 were postcardiac surgery patient populations and 1 was neurosurgical patients. Eight studies were included in the meta-analysis [Figure 1].

\section{Data extraction and statistical analysis}

Data were extracted independently by two authors and analyzed using Review Manager (RevMan) [Computer program]. Version 5.3. ${ }^{[14]}$ Copenhagen: The Nordic Cochrane Centre, The Cochrane Collaboration, 2014. For each study, the characteristics of the study, number of participants, characteristics of included patients, selection criteria, drug and dose used, and outcomes observed in the study were extracted. Most studies reported in-hospital mortality and 28 days mortality outcomes. Some studies reported 28 days mortality only and others reporting in-hospital mortality only. To include all RCTs in this meta-analysis, the authors have performed a separate analyses on both 28 days mortality and in-hospital mortality for this reason. All studies had adequate randomization and blinding. The quality of the RCTs was evaluated using the method described in the Cochrane Handbook for Systematic Reviews of Interventions. ${ }^{[15]}$ In each study, patients were given either a dose of statin (simvastatin 20/40/80 mg, rosuvastatin 40 mg or atorvastatin $20 \mathrm{mg}$ ) or a placebo. Low-dose statin was defined as simvastatin $20 \mathrm{mg}$ or atorvastatin $20 \mathrm{mg}$. High dose was defined as simvastatin $40 \mathrm{mg}$ or $80 \mathrm{mg}$ or rosuvastatin $40 \mathrm{mg} \cdot{ }^{[16,17]}$

\section{Results}

We examined a total of 8 RCTs with a total of 2275 patients. Figures 2 and 3 show forest plots of 28-day mortality and in-hospital mortality, respectively. Table 1 shows the 8 RCTs and the characteristics of each study. Table 2 shows assessment of bias for each

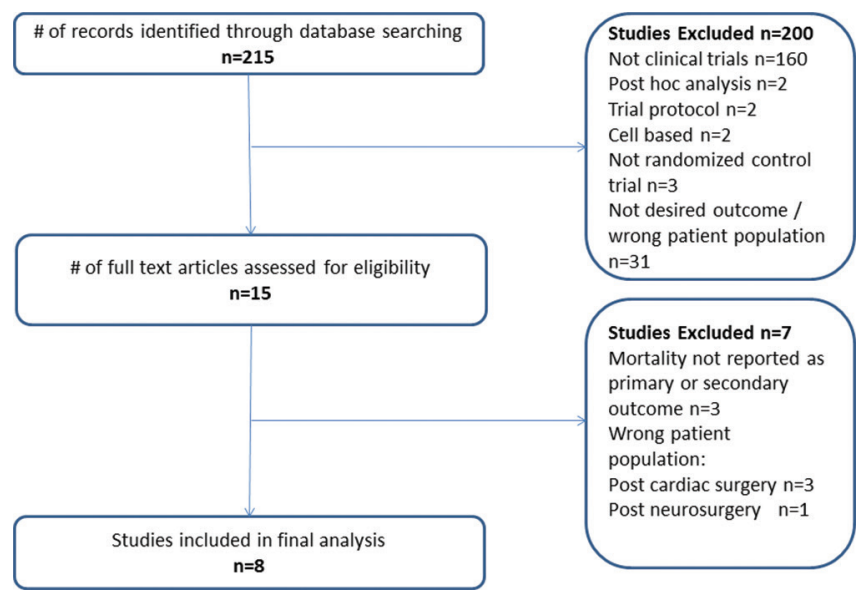

Figure I: Studies included in this meta-analysis 


\begin{tabular}{|c|c|c|c|c|c|c|c|c|}
\hline Study or Subgroup & $\begin{array}{r}\text { Stati } \\
\text { Events }\end{array}$ & & $\begin{array}{l}\text { Place } \\
\text { Events }\end{array}$ & Total & Weight & $\begin{array}{c}\text { Risk Ratio } \\
\text { M-H, Fixed, } 95 \% \mathrm{Cl}\end{array}$ & & $\begin{array}{c}\text { Risk Ratio } \\
\text { M.H, Fixed, } 95 \% \text { Cl }\end{array}$ \\
\hline Kruger 2013 & 12 & 123 & 22 & 127 & $18.5 \%$ & $0.56[0.29,1.09]$ & & $\rightarrow$ \\
\hline McAuley 2014 & 57 & 258 & 75 & 279 & $61.5 \%$ & $0.82[0.61,1.11]$ & & 난 \\
\hline Papazian 2013 & 31 & 146 & 21 & 138 & $18.4 \%$ & $1.40[0.84,2.31]$ & & 7 \\
\hline Patel 2012 & 2 & 49 & 2 & 51 & $1.7 \%$ & $1.04[0.15,7.10]$ & & \\
\hline Total (95\% Cl) & & 576 & & 595 & $100.0 \%$ & $0.88[0.70,1.12]$ & & 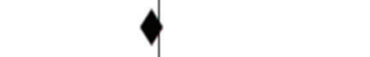 \\
\hline Total events & 102 & & 120 & & & & & \\
\hline $\begin{array}{l}\text { Heterogeneity Chi } \\
\text { Test for overall effect }\end{array}$ & $\begin{array}{l}5.22, \mathrm{df}= \\
Z=1.03\end{array}$ & $\begin{array}{l}3(P= \\
P=0.3\end{array}$ & $\begin{array}{l}0.16) ; r^{2}= \\
30)\end{array}$ & $43 \%$ & & & 0.01 & $\begin{array}{cccc}1 & 1 & 10 & 10 \\
\text { Favours Statin } & \text { Favours Placebo }\end{array}$ \\
\hline
\end{tabular}

Figure 2: Forest plot of proportional effect on 28-day mortality. Forest plot of proportional effect of all studies (low and high doses) on 28-day mortality (Diamonds $=$ totals and subtotals [95\% confidence interval]. Squares $=$ individual studies [horizontal lines are $95 \%$ confidence intervals]. Area of square proportional to amount of statistical information in that category. Risk ratios are weighted to represent heterogeneity)

\begin{tabular}{|c|c|c|c|c|c|c|c|c|c|c|}
\hline Study or Subgroup & Statin & in & Placebo & bo & Weight & \multirow{2}{*}{$\begin{array}{c}\begin{array}{c}\text { Risk Ratio } \\
\text { M-H, Fixed, } 95 \% \mathrm{Cl}\end{array} \\
1.00[0.59,1.70]\end{array}$} & \multicolumn{4}{|c|}{$\begin{array}{c}\text { Risk Ratio } \\
\text { M-H, Fixed, } 95 \% \mathrm{Cl}\end{array}$} \\
\hline Craig 2011 & 19 & 63 & 19 & 63 & $7.2 \%$ & & & & & \\
\hline Kruger 2011 & 6 & 75 & 4 & 75 & $1.5 \%$ & $1.50[0.44,5.10]$ & & & & \\
\hline Kruger 2013 & 16 & 123 & 23 & 127 & $8.6 \%$ & $0.72[0.40,1.29]$ & & & - & \\
\hline McAuley 2014 & 67 & 258 & 90 & 279 & $32.8 \%$ & $0.81[0.62,1.05]$ & & & & \\
\hline National Heart 2014 & 108 & 379 & 91 & 366 & $35.1 \%$ & $1.15[0.90,1.46]$ & & & $=$ & \\
\hline Novack 2009 & 0 & 42 & 0 & 41 & & Not estimable & & & & \\
\hline Papazian 2013 & 43 & 146 & 38 & 138 & $14.8 \%$ & $1.07[0.74,1.55]$ & & & & \\
\hline Patel 2012 & 0 & 0 & 0 & 0 & & Not estimable & & & & \\
\hline Total $(95 \% \mathrm{Cl})$ & & 1086 & & 1089 & $100.0 \%$ & $0.98[0.85,1.14]$ & & & & \\
\hline Total events & 259 & & 265 & & & & & & & \\
\hline $\begin{array}{l}\text { Heterogeneity: } \mathrm{Chi}^{2}= \\
\text { Test for overall effect: }\end{array}$ & $\begin{array}{l}.49, d f= \\
z=0.25(\end{array}$ & $\begin{array}{l}5(P=0 \\
=0.80\end{array}$ & 1.36); $1^{2}=$ & & & & 0.2 & $\begin{array}{c}0.5 \\
\text { Favours [Statin] }\end{array}$ & $\begin{array}{c}2 \\
\text { Favours [control] }\end{array}$ & 5 \\
\hline
\end{tabular}

Figure 3: Forest plot of proportional effect for in-hospital mortality of individual studies. Forest plot of proportional effect on in-hospital mortality (Diamonds $=$ totals and subtotals [ $95 \%$ confidence interval]. Squares $=$ individual studies [horizontal lines are $95 \%$ confidence intervals]. Area of square proportional to amount of statistical information in that category. Risk ratios are weighted to represent heterogeneity)

study. All studies used in the meta-analysis are listed in Appendix 1.

\section{All dose statin, 28-day mortality}

Results from 1171 patients demonstrated that there was no significant difference between statin $(102 / 576)$ and placebo $(120 / 595)$ for 28 day mortality (relative risk [RR] $0.8895 \%$ confidence interval [CI], 0.70-1.12 and $P=0.16$ ), with a heterogeneity of the trials $I^{2}=43 \%$, $P=0.16$ [Figure 2].

\section{All dose statin, in-hospital mortality}

Results from 2175 patients demonstrated that there was no significant difference between statin (259/1086) versus placebo $(265 / 1089)$ for in-hospital mortality (RR $0.9895 \% \mathrm{CI}, 0.85-1.14 P=0.36$ ) with low heterogeneity between the studies $I^{2}=9 \% P=0.36$. The authors excluded 83 patients from the Novack 2009 study from the analysis, as both arms of the trial had zero events [Figure 3]. ${ }^{[18]}$

\section{Low dose statin, in-hospital mortality}

Results from 400 patients demonstrated no statistically significant difference between low-dosage statin use $(22 / 198)$ versus placebo $(27 / 202)$ for in-hospital mortality [RR 0.81 CI (0.44-1.49) $P=0.27, I^{2}=16 \%$ Figure 4].

\section{High dose statin, in-hospital mortality}

Results from 1692 patients demonstrated that there was no significance between high-dose statin usage $(237 / 846)$ versus placebo $(239 / 846)$ for in-hospital mortality [RR $0.9995 \%$ CI $0.85-1.16 P=0.28, I^{2}=21 \%$, Figure 5].

\section{Adverse effects in high dose and low dose groups}

From all reported trials, patients with sepsis who received statins did not have a significantly higher incidence of adverse effects compared to placebo. In patients who were treated with high-dose statins, the incidence of adverse effects was higher $(11.6 \%$ vs. $8.5 \%, P>0.05)$, but this failed to reach statistical significance. 


\section{Table I: Characteristics of included studies}

\begin{tabular}{|c|c|c|c|c|c|c|}
\hline Study & Method & Participants & Interventions & $\begin{array}{l}\text { Low or } \\
\text { high } \\
\text { dose }\end{array}$ & Outcomes & $\begin{array}{c}\text { Number of } \\
\text { patients }\end{array}$ \\
\hline Kruger 20I |[27] & $\begin{array}{l}\text { Prospective } \\
\text { double-blind } \\
\text { placebo-controlled } \\
\text { RCT }\end{array}$ & $\begin{array}{l}\text { Patients with } 2 / 4 \text { SIRS criteria } \\
\text { present and known or suspected } \\
\text { infection, preexisting statin } \\
\text { therapy, and the treating physician } \\
\text { prepared to either continue or } \\
\text { discontinue this therapy }\end{array}$ & $\begin{array}{l}20 \text { mg atorvastatin or placebo } \\
\text { administered for the duration } \\
\text { of hospital admission up to a } \\
\text { maximum of } 28 \text { days }\end{array}$ & Low & $\begin{array}{l}\text { Progression of sepsis (SOFA } \\
\text { score), 28-day mortality, } \\
\text { requirement of ICU } \\
\text { admission, changes in } \\
\text { inflammatory markers, } \\
\text { changes in lipid profile }\end{array}$ & $150(75 / 75)$ \\
\hline Kruger $2013^{[28]}$ & $\begin{array}{l}\text { Prospective } \\
\text { double-blind } \\
\text { placebo-controlled } \\
\text { RCT }\end{array}$ & $\begin{array}{l}\text { Patients } 2 / 4 \text { SIRS criteria }+ \\
\text { known/suspected infection, } \\
\text { preexisting statin therapy, and } \\
\text { the treating physician prepared } \\
\text { to either continue or discontinue } \\
\text { this therapy }\end{array}$ & $\begin{array}{l}20 \text { mg atorvastatin or placebo } \\
\text { administered for the duration } \\
\text { of hospital admission up to a } \\
\text { maximum of } 28 \text { days }\end{array}$ & Low & $\begin{array}{l}\text { Progression of sepsis (SOFA } \\
\text { score), 28-day mortality, } \\
\text { requirement of ICU } \\
\text { admission, changes in } \\
\text { inflammatory markers, } \\
\text { changes in lipid profile }\end{array}$ & $150(75 / 75)$ \\
\hline Craig 20 I | [29] & $\begin{array}{l}\text { Single-center, } \\
\text { prospective, } \\
\text { double-blind, } \\
\text { placebo-controlled } \\
\text { RCT }\end{array}$ & $\begin{array}{l}\text { Patients undergoing mechanical } \\
\text { ventilation in ITU within } 48 \mathrm{~h} \text { of } \\
\text { the diagnosis of ALI and ARDS }\end{array}$ & $\begin{array}{l}80 \mathrm{mg} \text { simvastatin or placebo } \\
\text { was administered daily for } \\
\text { up to } 14 \text { days if the CK } \\
<10 \times \text { reference range and } \\
\text { transaminases }<3 \times \text { upper } \\
\text { normal range. Treatment } \\
\text { was continued until death, } \\
\text { discontinuation of mechanical } \\
\text { ventilation, request by } \\
\text { patient/relative or on day } 14\end{array}$ & High & $\begin{array}{l}\text { Extravascular lung water, } \\
\text { ventilator-free days, } \\
\text { duration of mechanical } \\
\text { ventilation, ICU-free days, } \\
\text { ICU survival, hospital length } \\
\text { of stay, hospital survival }\end{array}$ & $540(270 / 270)$ \\
\hline $\begin{array}{l}\text { National Heart } \\
\text { Lung and Blood } \\
\text { Institute } 2014^{[13]}\end{array}$ & $\begin{array}{l}\text { Multicenter, } \\
\text { double blind, } \\
\text { placebo controlled } \\
\text { RCT }\end{array}$ & $\begin{array}{l}\text { Patients who were undergoing } \\
\text { mechanical ventilation with the } \\
\text { presence of bilateral pulmonary } \\
\text { infiltrates on chest X-ray, and } \\
\text { a known or suspected source } \\
\text { of infection (WCC }>12 \text { or }<4 \text {, } \\
>10 \% \text { WCC differential, or } \\
\text { temperature }>38 \text { or }<36 \text { ) }\end{array}$ & $\begin{array}{l}40 \mathrm{mg} \text { loading dose of either } \\
\text { rosuvastatin or placebo given } \\
\text { orally, then } 20 \mathrm{mg} \text { thereafter } \\
\text { until the } 3^{\text {rd }} \text { day from } \\
\text { discharge from ITU, day } 28 \text {, } \\
\text { hospital discharge or death, } \\
\text { which-ever came first }\end{array}$ & High & $\begin{array}{l}\text { In-hospital mortality before } \\
\text { discharge, day- } 60 \text { mortality, } \\
\text { ventilator-free days, organ } \\
\text { failure free days to day } 14 \text {, } \\
\text { ICU free days to day } 28\end{array}$ & $745(379 / 366)$ \\
\hline McAuley $20 \mid 4^{[23]}$ & $\begin{array}{l}\text { Multicenter, } \\
\text { double blind, } \\
\text { placebo controlled } \\
\text { RCT }\end{array}$ & $\begin{array}{l}\text { Patients > I } 6 \text { years requiring } \\
\text { mechanical ventilation with ARDS } \\
\text { within } 48 \text { h of onset of ARDS. } \\
\text { ARDS defined as the ration of } \\
\text { partial pressure of oxygen to the } \\
\mathrm{FiO}_{2} \text {, with bilateral pulmonary } \\
\text { infiltrates on chest X-ray }\end{array}$ & $\begin{array}{l}80 \mathrm{mg} \text { of simvastatin via } \\
\text { nasogastric tube daily or } \\
\text { placebo for until day } 28 \text { or } \\
\text { discharge from critical care }\end{array}$ & High & $\begin{array}{l}\text { Ventilator-free days, days } \\
\text { free of nonpulmonary organ } \\
\text { failure, } 28 \text { days mortality, } \\
\text { in-hospital mortality, in } \\
\text { critical care mortality }\end{array}$ & $540(259 / 281)$ \\
\hline Novack 2009 ${ }^{[18]}$ & $\begin{array}{l}\text { Single-center, } \\
\text { double-blinded, } \\
\text { placebo controlled } \\
\text { RCT }\end{array}$ & $\begin{array}{l}\text { Patients }>18 \text { years, not receiving } \\
\text { statin therapy during the } 3 \text { months } \\
\text { prior to admission, within } 12 \mathrm{~h} \\
\text { of admission to general medical } \\
\text { ward, have a documented bacterial } \\
\text { infection and have intravenous } \\
\text { antibiotics prescribed (independent } \\
\text { of study team) }\end{array}$ & $\begin{array}{l}40 \mathrm{mg} \text { simvastatin or placebo } \\
\text { given orally immediately } \\
\text { postenrollment followed } \\
\text { by } 20 \mathrm{mg} \text { daily until } \\
\text { hospital discharge or the } \\
\text { development of severe sepsis }\end{array}$ & High & $\begin{array}{l}\text { Development of severe } \\
\text { sepsis (defined by } \\
\text { PROWESSS investigators), } \\
\text { change in level of cytokines, } \\
\text { death, length of stay, need } \\
\text { for mechanical ventilation }\end{array}$ & $83(42 / 4 I)$ \\
\hline Papazian 20I $3^{[12]}$ & $\begin{array}{l}\text { Multicenter trial } \\
\text { placebo-controlled, } \\
\text { double-blind, } \\
\text { parallel-group RCT }\end{array}$ & $\begin{array}{l}\text { Patients who required mechanical } \\
\text { ventilation for more than } 2 \text { days } \\
\text { and who had a suspected } \\
\text { ventilator acquired pneumonia, } \\
\text { defined as a modified clinical } \\
\text { pulmonary infection score of } 5 \text { or } \\
\text { greater }\end{array}$ & $\begin{array}{l}60 \text { mg statin or placebo given } \\
\text { orally from study inclusion to } \\
\text { ICU discharge, death, or day } \\
28, \text { whichever occurred first }\end{array}$ & High & $\begin{array}{l}\text { 28-day mortality, } \\
\text { day-I } 4 \text { mortality, ICU } \\
\text { mortality, ICU-free days, } \\
\text { ventilator-free days }\end{array}$ & $284(146 / 136)$ \\
\hline Patel 20I $2^{[19]}$ & $\begin{array}{l}\text { A single-center } \\
\text { phase II, } \\
\text { double-blind } \\
\text { placebo-controlled } \\
\text { RCT }\end{array}$ & $\begin{array}{l}\text { Patients age }>18 \text { years with } \\
\text { documented new or suspected } \\
\text { infection with } 2 \text { or more SIRS } \\
\text { criteria for }<24 \mathrm{~h}\end{array}$ & $\begin{array}{l}40 \text { mg atorvastatin or placebo } \\
\text { administered within } 24 \text { h of } \\
\text { randomization and continued } \\
\text { until discharge or day } 28 \text {, } \\
\text { whichever occurred first }\end{array}$ & High & $\begin{array}{l}\text { Progression of sepsis to } \\
\text { severe sepsis (identified } \\
\text { using the SSCG screening } \\
\text { tool), ITU admission rate, } \\
\text { hospital readmission rate at } \\
28 \text { days and I year, length } \\
\text { of stay, and } 28 \text {-day mortality } \\
\text { and I-year mortality }\end{array}$ & $100(49 / 5 I)$ \\
\hline
\end{tabular}

RCT: Randomized controlled trial; SIRS: Systemic inflammatory response syndrome; SSCG: Surviving Sepsis Campaign Guidelines; ICU: Intensive Care Unit; WCC: White cell count; ARDS: Acute respiratory distress syndrome; ALI: Acute lung injury; CK: Creatinine kinase; SOFA: Sequential organ failure assessment; ITU: Intensive treatment unit 


\section{Table 2: Assessment of risk of bias}

\begin{tabular}{|c|c|c|c|c|c|c|}
\hline \multirow[t]{2}{*}{ Study } & \multicolumn{6}{|c|}{ Type of bias } \\
\hline & Randomization & $\begin{array}{l}\text { Allocation } \\
\text { concealment }\end{array}$ & $\begin{array}{l}\text { Blinding of } \\
\text { participants and } \\
\text { personnel }\end{array}$ & $\begin{array}{l}\text { Blinding of } \\
\text { outcome }\end{array}$ & $\begin{array}{l}\text { Incomplete } \\
\text { outcome data }\end{array}$ & Reporting bias \\
\hline Craig 20I |[29] & $\begin{array}{l}\text { Low risk } \\
\text { Randomization } \\
\text { performed by } \\
\text { independent } \\
\text { statistician }\end{array}$ & $\begin{array}{l}\text { Low risk } \\
\text { Independent clinical } \\
\text { trial pharmacist } \\
\text { performed treatment } \\
\text { randomization }\end{array}$ & $\begin{array}{l}\text { Low risk } \\
\text { Study drugs were } \\
\text { encapsulated } \\
\text { by independent } \\
\text { pharmacist }\end{array}$ & $\begin{array}{l}\text { Low risk } \\
\text { Double-blind }\end{array}$ & $\begin{array}{l}\text { Low risk } \\
\text { None lost to } \\
\text { follow-up }\end{array}$ & $\begin{array}{l}\text { Low risk } \\
\text { Study protocol } \\
\text { published prior to } \\
\text { commencement } \\
\text { of trial and all } \\
\text { outcomes reported }\end{array}$ \\
\hline Kruger 20I |[27] & $\begin{array}{l}\text { Low risk } \\
\text { Computer-generated } \\
\text { randomization }\end{array}$ & $\begin{array}{l}\text { Low risk } \\
\text { Placed in sealed } \\
\text { opaque envelopes }\end{array}$ & $\begin{array}{l}\text { Low risk } \\
\text { Study drugs were } \\
\text { encapsulated } \\
\text { by independent } \\
\text { pharmacist }\end{array}$ & $\begin{array}{l}\text { Low risk } \\
\text { Double-blind }\end{array}$ & $\begin{array}{l}\text { Low risk } \\
\text { 2/75 missing } \\
\text { from treatment } \\
\text { group. Reasons } \\
\text { for withdrawal } \\
\text { acceptable and } \\
\text { documented }\end{array}$ & \\
\hline Kruger 201 $3^{[28]}$ & $\begin{array}{l}\text { Randomization was } \\
\text { performed using a } \\
\text { computer-generated } \\
\text { list }\end{array}$ & $\begin{array}{l}\text { Low risk } \\
\text { Allocation concealed } \\
\text { using computer }\end{array}$ & $\begin{array}{l}\text { Low risk } \\
\text { Placebo/study } \\
\text { drug identical. } \\
\text { Both prepared by } \\
\text { central pharmacy } \\
\text { under controlled } \\
\text { conditions }\end{array}$ & $\begin{array}{l}\text { Low risk } \\
\text { Double-blind }\end{array}$ & $\begin{array}{l}\text { Low risk } \\
32 \text { patients ( } 15 \\
\text { treatment/ } 7 \\
\text { placebo) dropped } \\
\text { out from study } \\
\text { due to adverse } \\
\text { effects of drug }\end{array}$ & \\
\hline $\begin{array}{l}\text { National Heart } \\
\text { Lung and Blood } \\
\text { Institute } 2014^{[13]}\end{array}$ & $\begin{array}{l}\text { Patients were } \\
\text { randomly assigned in } \\
\text { permuted blocks of } 8\end{array}$ & $\begin{array}{l}\text { Unclear risk } \\
\text { Insufficient information } \\
\text { to permit judgment }\end{array}$ & $\begin{array}{l}\text { Unclear risk } \\
\text { Reported as } \\
\text { double blind } \\
\text { but no method } \\
\text { described }\end{array}$ & $\begin{array}{l}\text { Low risk } \\
\text { Blinding of outcome } \\
\text { assessment to } \\
\text { all participants } \\
\text { except pharmacist, } \\
\text { lab technicians } \\
\text { and leaders of } \\
\text { coordinating center }\end{array}$ & $\begin{array}{l}\text { Low risk } \\
\text { No missing } \\
\text { outcome data. } \\
\text { Study stopped } \\
\text { early due to } \\
\text { futility }\end{array}$ & $\begin{array}{l}\text { Low risk } \\
\text { Study protocol } \\
\text { is available and } \\
\text { all of the study's } \\
\text { pre-specified } \\
\text { (primary and } \\
\text { secondary) } \\
\text { outcomes that are } \\
\text { of interest in the } \\
\text { review have been } \\
\text { reported }\end{array}$ \\
\hline McAuley $2014^{[23]}$ & $\begin{array}{l}\text { Low risk } \\
\text { Centralized } \\
\text { computer-generated } \\
\text { randomization } \\
\text { available } 24 \mathrm{~h} / \text { day }\end{array}$ & $\begin{array}{l}\text { Low risk } \\
\text { Computer-assigned } \\
\text { centralized block } \\
\text { randomization I:I ratio }\end{array}$ & $\begin{array}{l}\text { Low risk } \\
\text { Double-blind } \\
\text { and study drugs } \\
\text { reported as } \\
\text { identical }\end{array}$ & $\begin{array}{l}\text { Low risk } \\
\text { Double-blind }\end{array}$ & $\begin{array}{l}\text { Low risk } \\
3 / 540 \text { lost to } \\
\text { follow-up } \\
8 / 540 \text { did not } \\
\text { receive study } \\
\text { drug }\end{array}$ & $\begin{array}{l}\text { Low risk } \\
\text { Study protocol } \\
\text { published before } \\
\text { clinical trial and all } \\
\text { outcomes have been } \\
\text { reported }\end{array}$ \\
\hline Novack 2009[18] & $\begin{array}{l}\text { Low risk } \\
\text { Computer-generated } \\
\text { randomization }\end{array}$ & $\begin{array}{l}\text { Low risk } \\
\text { Allocation concealment } \\
\text { was ensured through } \\
\text { use of sequentially } \\
\text { numbered envelopes }\end{array}$ & $\begin{array}{l}\text { Low risk } \\
\text { Study drugs } \\
\text { or placebo } \\
\text { encapsulated } \\
\text { and prepared by } \\
\text { pharmacy }\end{array}$ & $\begin{array}{l}\text { Low risk } \\
\text { Double-blind }\end{array}$ & $\begin{array}{l}\text { Low risk } \\
\text { No losses to } \\
\text { follow-up }\end{array}$ & \\
\hline Papazian $2013^{[12]}$ & $\begin{array}{l}\text { Low risk } \\
\text { Computer-generated } \\
\text { randomization using } \\
\text { table in blocks of } 4\end{array}$ & $\begin{array}{l}\text { Low risk } \\
\text { Block size unknown } \\
\text { to investigators, } \\
\text { telephone-based } \\
\text { randomization }\end{array}$ & $\begin{array}{l}\text { Low risk } \\
\text { Study drug } \\
\text { identical to } \\
\text { placebo, } \\
\text { investigators and } \\
\text { patients blinded }\end{array}$ & $\begin{array}{l}\text { Low risk } \\
\text { Double-blind }\end{array}$ & $\begin{array}{l}\text { Low risk } \\
\text { I6 patients } \\
\text { excluded from } \\
\text { analysis ( } 7 \\
\text { treatment/9 } \\
\text { placebo), reasons } \\
\text { for withdrawal } \\
\text { appropriate and } \\
\text { well documented }\end{array}$ & $\begin{array}{l}\text { Low risk } \\
\text { Primary and } \\
\text { secondary outcomes } \\
\text { reported in full }\end{array}$ \\
\hline Patel $2012^{[19]}$ & $\begin{array}{l}\text { Low risk } \\
\text { Computer-generated } \\
\text { randomization } \\
\text { sequence in blocks } \\
\text { of } 4\end{array}$ & $\begin{array}{l}\text { Low risk } \\
\text { Sequentially numbered } \\
\text { identical drug } \\
\text { containers }\end{array}$ & $\begin{array}{l}\text { Low risk } \\
\text { Study drugs } \\
\text { prepared by } \\
\text { independent } \\
\text { pharmacy and } \\
\text { investigators } \\
\text { blinded }\end{array}$ & $\begin{array}{l}\text { Low risk } \\
\text { Double-blind }\end{array}$ & $\begin{array}{l}\text { Low risk } \\
\text { No patients lost } \\
\text { to follow-up }\end{array}$ & $\begin{array}{l}\text { Low risk } \\
\text { The study protocol } \\
\text { is available and } \\
\text { all of the study's } \\
\text { prespecified (primary } \\
\text { and secondary) } \\
\text { outcomes that are } \\
\text { of interest in the } \\
\text { review have been } \\
\text { reported in the } \\
\text { prespecified way }\end{array}$ \\
\hline
\end{tabular}




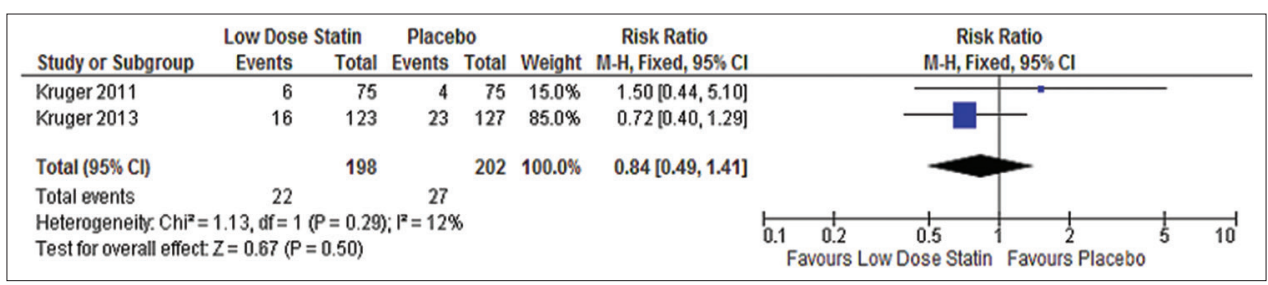

Figure 4: Forest plot of proportional effect for low dose statin, in-hospital mortality. Forest plot of proportional effect on in-hospital mortality (Diamonds = totals and subtotals [95\% confidence interval]. Squares = individual studies [horizontal lines are $95 \%$ confidence intervals]. Area of square proportional to amount of statistical information in that category. Risk ratios are weighted to represent heterogeneity)

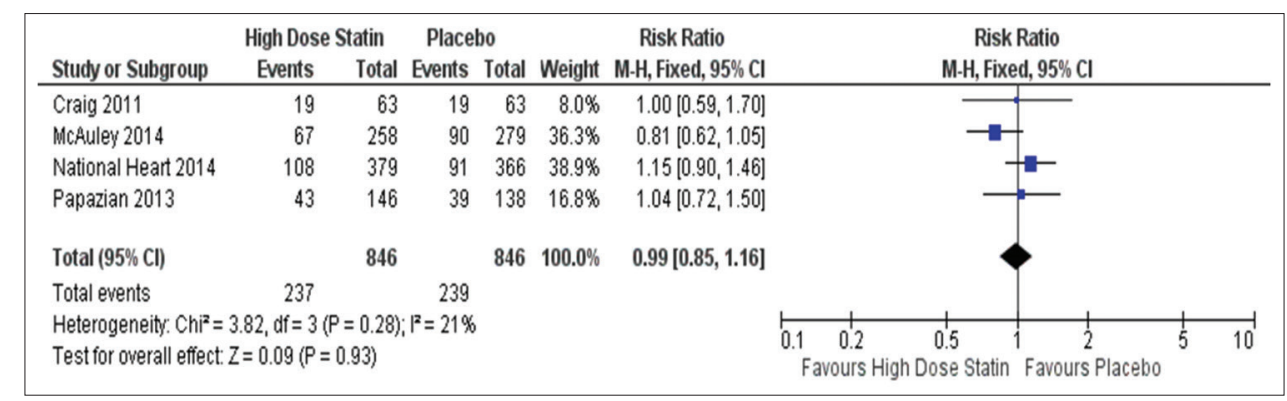

Figure 5: Forest plot of proportional effect for high dose statin, in-hospital mortality. Forest plot of proportional effect on in-hospital mortality (Diamonds $=$ totals and subtotals [ $95 \%$ confidence interval]. Squares = individual studies [horizontal lines are $95 \%$ confidence intervals]. Area of square proportional to amount of statistical information in that category. Risk ratios are weighted to represent heterogeneity)

\section{Discussion}

This meta-analysis demonstrated that statin therapy does not reduce in-hospital or 28 days mortality in sepsis when compared to placebo. This meta-analysis also failed to show any significant difference in outcome of treating patients with low or high dose statins.

Statins were shown to be beneficial in animal model studies, ${ }^{[6]}$ and early RCTs demonstrated benefits in critically ill patients with sepsis, however these studies were single center RCTs with a relatively small sample size. ${ }^{[19]}$ In this study, we investigated whether the dose of statin was associated with adverse events. Statin therapy is associated with musculoskeletal side effects including myopathy, myositis, and rhabdomyolysis. Myalgia and arthralgia are reported in up to $5 \%$ of participants in clinical trials. ${ }^{[20-22]}$ Reported incidence of dose dependent adverse events such as deranged hepatic transaminases was $0.5-2 \% .{ }^{[16,20]}$ In one study, there were a significant number of adverse events related to the intervention arm when using $80 \mathrm{mg}$ of simvastatin. ${ }^{[23]}$ However, across all studies in this analysis, the total incidence of adverse events in patients who were treated with high dose statins was not significantly different compared to placebo or to low dose statins.

In this up to date meta-analysis, we focused only on eligible RCTs to eliminate the risk of confounding variables often seen in observation studies. Compared to previous meta-analyses, we have not only included up to date RCTs but also tried to delineate the role of high and low-dose statins. ${ }^{[24]}$ These findings are in contradiction to previous literature reviews and observational studies. ${ }^{[7,25]}$ As our meta-analysis relies purely on RCTs, this limits the study to a relatively small sample size. This is particularly true for the low-dose statin subgroup of patients.

While preclinical and observational studies hypothesized the potential benefits of statin usage in sepsis, our meta-analysis contradicts this presumption. Although the beneficial effects in vitro and animal models have been well documented in literature, ${ }^{[6,11]}$ these effects do not confer a detectable benefit in large human model RCTs. This could be due to the heterogeneity of the populations being studied; there is an inter subject variability in the host response due to age, existing comorbidities and genetic profile. Genes involved in sepsis have been analyzed for links between single-nucleoside polymorphisms and sepsis susceptibility, organ dysfunction and mortality. ${ }^{[26]}$ Despite the heterogeneity of the populations being studied, there are no suggestions from this study that a specific population group would benefit from statin therapy. 


\section{Conclusions}

This meta-analysis found no beneficial effect of statin therapy in the context of sepsis and the mortality of critically ill patients. Our findings are in contradiction to previous reviews of the literature and also to those of observational studies. ${ }^{[7,19,25]}$

\section{Financial support and sponsorship}

Dr. Veenith is supported by NIAA clinical research training fellowship and Beverly Sackler Studentship.

\section{Conflicts of interest}

\section{There are no conflicts of interest.}

\section{References}

1. Vincent JL, Sakr Y, Sprung CL, Ranieri VM, Reinhart K, Gerlach H, et al. Sepsis in European intensive care units: Results of the SOAP study. Crit Care Med 2006;34:344-53.

2. Hall MJ, Williams SN, DeFrances CJ, Golosinskiy A. Inpatient care for septicemia or sepsis: A challenge for patients and hospitals. NCHS Data Brief 2011;62:1-8

3. Torio CM, Andrews RM. National Inpatient Hospital Costs: The Most Expensive Conditions by Payer, 2011: Statistical Brief \#160. Healtheare Cost and Utilization Project (HCUP) Statistical Briefs; 2006. Available from: http://www.ncbi.nlm.nih.gov/pubmed/24199255. [Last cited on $2016 \mathrm{Jul} 24$ ].

4. Terblanche M, Almog Y, Rosenson RS, Smith TS, Hackam DG. Statins: Panacea for sepsis? Lancet Infect Dis 2006;6:242-8.

5. Liao JK, Laufs U. Pleiotropic effects of statins. Annu Rev Pharmacol Toxicol 2005;45:89-118.

6. Merx MW, Liehn EA, Graf J, van de Sandt A, Schaltenbrand M, Schrader J, et al. Statin treatment after onset of sepsis in a murine model improves survival. Circulation 2005;112:117-24.

7. Kruger P, Fitzsimmons K, Cook D, Jones M, Nimmo G. Statin therapy is associated with fewer deaths in patients with bacteraemia. Intensive Care Med 2006;32:75-9.

8. Terblanche M, Almog Y, Rosenson RS, Smith TS, Hackam DG. Statins and sepsis: Multiple modifications at multiple levels. Lancet Infect Dis 2007;7:358-68.

9. Drage SM, Barber VS, Young JD. Statins and sepsis: Panacea or pandora's box? Lancet Infect Dis 2007;7:80.

10. De Loecker I, Preiser JC. Statins in the critically ill. Ann Intensive Care $2012 ; 2: 19$.

11. Greenwood J, Mason JC. Statins and the vascular endothelial inflammatory response. Trends Immunol 2007;28:88-98.

12. Papazian L, Roch A, Charles PE, Penot-Ragon C, Perrin G, Roulier $\mathrm{P}$, et al. Effect of statin therapy on mortality in patients with ventilator-associated pneumonia: A randomized clinical trial. JAMA 2013;310:1692-700.
13. Heart TN. Rosuvastatin for sepsis-associated acute respiratory distress syndrome. N Engl J Med 2014;370:2191-200.

14. The Cochrane Collaboration. Review Manager (RevMan) [Computer Program]. Copenhagen: The Nordic Cochrane Centre; 2014.

15. Higgins JPT, Green S, editors. Cochrane handbook for systematic reviews of interventions version 5.1.0 [updated March 2011]. The Cochrane Collaboration; 2011. Available from http://handbook. cochrane.org. [Last accessed on 2016 Sep 07].

16. Hsu I, Spinler SA, Johnson NE. Comparative evaluation of the safety and efficacy of HMG-CoA reductase inhibitor monotherapy in the treatment of primary hypercholesterolemia. Ann Pharmacother $1995 ; 29: 743-59$.

17. Jones PH, Davidson MH, Stein EA, Bays HE, McKenney JM, Miller $\mathrm{E}$, et al. Comparison of the efficacy and safety of rosuvastatin versus atorvastatin, simvastatin, and pravastatin across doses (STELLAR* Trial). Am J Cardiol 2003:92:152-60.

18. Novack V, Eisinger M, Frenkel A, Terblanche M, Adhikari NK, Douvdevani A, et al. The effects of statin therapy on inflammatory cytokines in patients with bacterial infections: A randomized doubleblind placebo controlled clinical trial. Intensive Care Med 2009;35:125560.

19. Patel JM, Snaith C, Thickett DR, Linhartova L, Melody T, Hawkey $\mathrm{P}$, et al. Randomized double-blind placebo-controlled trial of $40 \mathrm{mg} /$ day of atorvastatin in reducing the severity of sepsis in ward patients (ASEPSIS Trial). Crit Care 2012;16:R231.

20. Bradford RH, Shear CL, Chremos AN, Dujovne C, Downton M, Franklin FA, et al. Expanded clinical evaluation of lovastatin (EXCEL) study results. I. Efficacy in modifying plasma lipoproteins and adverse event profile in 8245 patients with moderate hypercholesterolemia. Arch Intern Med 1991;151:43-9.

21. Angulo P. Nonalcoholic fatty liver disease. N Engl J Med 2002;346:1221-31.

22. Farmer JA. Learning from the cerivastatin experience. Lancet 2001;358:1383-5.

23. McAuley DF, Laffey JG, O'Kane CM, Perkins GD, Mullan B, Trinder T.J, et al. Simvastatin in the acute respiratory distress syndrome. N Engl J Med 2014;371:1695-703.

24. Deshpande A, Pasupuleti V, Rothberg MB. Statin therapy and mortality from sepsis: A meta-analysis of randomized trials. Am J Med 2015;128:410-7.e1.

25. Almog Y, Shefer A, Novack V, Maimon N, Barski L, Eizinger M, et al. Prior statin therapy is associated with a decreased rate of severe sepsis. Circulation 2004;110:880-5.

26. Dhas BB, Ashmi H, Bhat BV. Sepsis genomies: Stepping forward toward sepsis prevention?. Int J Adv Med Health Res 2014;1:8-15.

27. Kruger PS, Harward ML, Jones MA, Joyce CJ, Kostner KM, Roberts MS, et al. Continuation of statin therapy in patients with presumed infection: A randomized controlled trial. Am J Respir Crit Care Med 2011;183:774-81.

28. Kruger P, Bailey M, Bellomo R, Cooper DJ, Harward M, Higgins A, et al. A multicenter randomized trial of atorvastatin therapy in intensive care patients with severe sepsis. Am J Respir Crit Care Med 2013;187:743-50.

29. Craig TR, Duffy MJ, Shyamsundar M, MeDowell C, O'Kane CM, Elborn JS, et al. A randomized clinical trial of hydroxymethylglutarylcoenzyme a reductase inhibition for acute lung injury (The HARP Study). Am J Respir Crit Care Med 2011;183:620-6. 


\section{Appendix}

\section{Appendix 1: Studies included in this meta-analysis}

1. Papazian L, Roch A, Charles PE, Penot-Ragon C, Perrin G, Roulier P, et al. Effect of statin therapy on mortality in patients with ventilator-associated pneumonia: A randomized clinical trial. JAMA 2013;310:1692-700.

2. National Heart, Lung, and Blood Institute ARDS Clinical Trials Network, Truwit JD, Bernard GR, Steingrub J, et al. Rosuvastatin for sepsis-associated acute respiratory distress syndrome. N Engl J Med 2014;370:2191-200.

3. Patel JM, Snaith C, Thickett DR, Linhartova L, Melody T, Hawkey P, et al. Randomized double-blind placebo-controlled trial of $40 \mathrm{mg}$ / day of atorvastatin in reducing the severity of sepsis in ward patients (ASEPSIS Trial). Crit Care 2012;16:R231.

4. McAuley DF, Laffey JG, O'Kane CM, Perkins GD, Mullan B, Trinder TJ, et al. Simvastatin in the acute respiratory distress syndrome. N Engl J Med 2014;371:1695-703.

5. Kruger PS, Harward ML, Jones MA, Joyce CJ, Kostner KM, Roberts MS, et al. Continuation of statin therapy in patients with presumed infection: A randomized controlled trial. Am J Respir Crit Care Med 2011;183:774-81.

6. Kruger P, Bailey M, Bellomo R, Cooper DJ, Harward M, Higgins A, et al. A multicenter randomized trial of atorvastatin therapy in intensive care patients with severe sepsis. Am J Respir Crit Care Med 2013;187:743-50.

7. Craig TR, Duffy MJ, Shyamsundar M, McDowell C, O'Kane CM, Elborn JS, et al. A randomized clinical trial of hydroxymethylglutaryl- coenzyme a reductase inhibition for acute lung injury (The HARP Study). Am J Respir Crit Care Med 2011;183:620-6.

8. Novack V, Eisinger M, Frenkel A, Terblanche M, Adhikari NK, Douvdevani A, et al. The effects of statin therapy on inflammatory cytokines in patients with bacterial infections: A randomized double-blind placebo controlled clinical trial. Intensive Care Med 2009;35:1255-60.

\section{Search criteria}

(("sepsis" [MeSH Terms] OR "sepsis"[All Fields]) AND ((("atorvastatin"[Supplementary Concept] OR “atorvastatin"[All Fields]) OR ("rosuvastatin"[Supplementary Concept] OR "rosuvastatin"[All Fields])) OR ("simvastatin" [MeSH Terms] OR "simvastatin"[All Fields]))) OR (("intensive care"[MeSH Terms] OR ("intensive" [All Fields] AND "care"[All Fields]) OR “intensive care"[All Fields]) AND ((("atorvastatin"[Supplementary Concept] OR "atorvastatin"[All Fields]) OR ("rosuvastatin" [Supplementary Concept] OR "rosuvastatin"[All Fields])) OR ("simvastatin" [MeSH Terms] OR "simvastatin"[All Fields]))) 